\title{
Quantum Hyper-CPHASE gates with Polarisation and Orbital Angular Momentum Degrees of Freedom and Generalisation to Arbitrary Hyper-Conditional Gates
}

\author{
Mrittunjoy Guha Majumdar* \\ Department of Physics and Astrophysics, University of Delhi, Delhi - 110007
}

(Dated: May 17, 2020)

\begin{abstract}
In this paper, a controlled-phase-flip (P-CPF) gate using the polarisation and orbital angular momentum degrees of freedom for single-photon two-qubit quantum logic is proposed. This is critical to the realisation of quantum cluster states and graph networks using transverse degrees of freedom. A generalisation of the proposed scheme to arbitrary number and kinds of degrees of freedom, for optical systems, as well as arbitrary operations to be conditionally performed is proposed.
\end{abstract}

Keywords: Entanglement, Quantum Operation, Cluster States

Universal quantum information processing can be performed with single-qubit rotations and the two-qubit controlled-NOT operations, among other possible sets of universal generators of quantum gates $[1,2]$. Quantum information processing in optical systems, using photonic qubits and qumodes, have been realised for gate-based as well as measurement-based quantum computation and communication [3-7]. In optical systems, single-qubit operations as well as controlled logic operations over two or more qubits can be realised using linear optical elements such as beam-splitters and waveplates [8-11]. Quantum information processing can be done over multiple transverse degrees of freedom in optical systems, using hyper-entanglement and hybrid-entanglement $[12,13]$. This allows efficient computation and communication using multiple logical qubits over a lesser number of physical qubits. Measurement-based quantum computation has been realised in optical systems $[14,15]$, with Yokoyama et al multiplexing generating and characterised a continuous-variable cluster state containing more than 10,000 entangled modes [16], and Larsen et al recently proposing a scheme to generate more than 30,000 entangled modes in a two-dimensional cluster state [17].

In cluster-state quantum computing, the role of the Controlled-PHASE (CPHASE) or Controlled-PhaseFlip (CFP), as an entangler, is primary [18, 19]. In optical systems with multiple degrees of freedom, the realisation of a CPHASE gate has previously been undertaken, between polarisation and momentum degrees of freedom, thereby helping create a high fidelity four-qubit linear cluster state [20]. Cluster states have been created using the simultaneous entanglement of photons in three degrees of freedom (polarisation and double longitudinal momentum), in a hybrid approach to one-way quantum computing [13], while recently, an 18-qubit Greenberger-Horne-Zeilinger (GHZ) entangled state was experimentally demonstrated by simulta-

*mrittunjoy.guhamajumdar@cantab.net neously exploiting three different degrees of freedom (polarisation, path and orbital angular momentum) of six photons [21]. With regards to a single degree of freedom. Zou et al proposed a linear optical scheme for direct implementation of a nondestructive $\mathrm{N}$-qubit controlled phase (CPHASE) gate on the polarisation degree of freedom [22]. Lemr et al experimentally realized an optimal linear-optical controlled phase shift gate for arbitrary phases [23]. Controlled phase shift and CPHASE gates have been implemented using quantum dots and optical cavities [24-27].

In this work, a scheme for a conditional phase-flip $(\mathrm{CPF})$ gate using the polarisation and orbital angular momentum degrees of freedom is proposed. The scheme utilises linear as well as non-linear optical elements (such as a polarising beam splitter like a Wollaston prism), and is a fundamental step towards the realisation of one-time measurement-based quantum computation using optical systems. A generalisation of this scheme to arbitrary number and kinds of degrees of freedom as well as arbitrary operation to be conditionally performed is proposed, for optical systems.

\section{REALISATION OF HYPER-CPHASE GATE BETWEEN POLARISATION AND OAM DEGREES OF FREEDOM}

The scheme utilises the state discrimination for the different degrees of freedom (DOF) performed by a DOFspecific beam splitter, to selectively introduce a phaseflip operation in the other degree of freedom. In the fundamental unit of the Hyper-CPHASE using polarisation and orbital angular momentum degrees of freedom, polarisation beam splitters (PBS) for polarisation control and OAM-based beam splitters formed of MachZehnder interferometer with two Dove prisms for OAMcontrol are used. If we consider polarisation-controlled Hyper-CPHASE and an initial quantum state $\left|\psi_{i n}\right\rangle=$ $|0\rangle\left|E_{(0)}\right\rangle+|1\rangle\left|E_{(1)}\right\rangle$, where $|i\rangle(i=0,1)$ are the polarisation states while $\left|E_{(i)}\right\rangle(i=0,1)$ are the orbital angular 
momentum states in the input quantum state that are associated with corresponding $|i\rangle$ polarisation states.

\section{GENERALISATION OF HYPER-CONDITIONAL GATES}

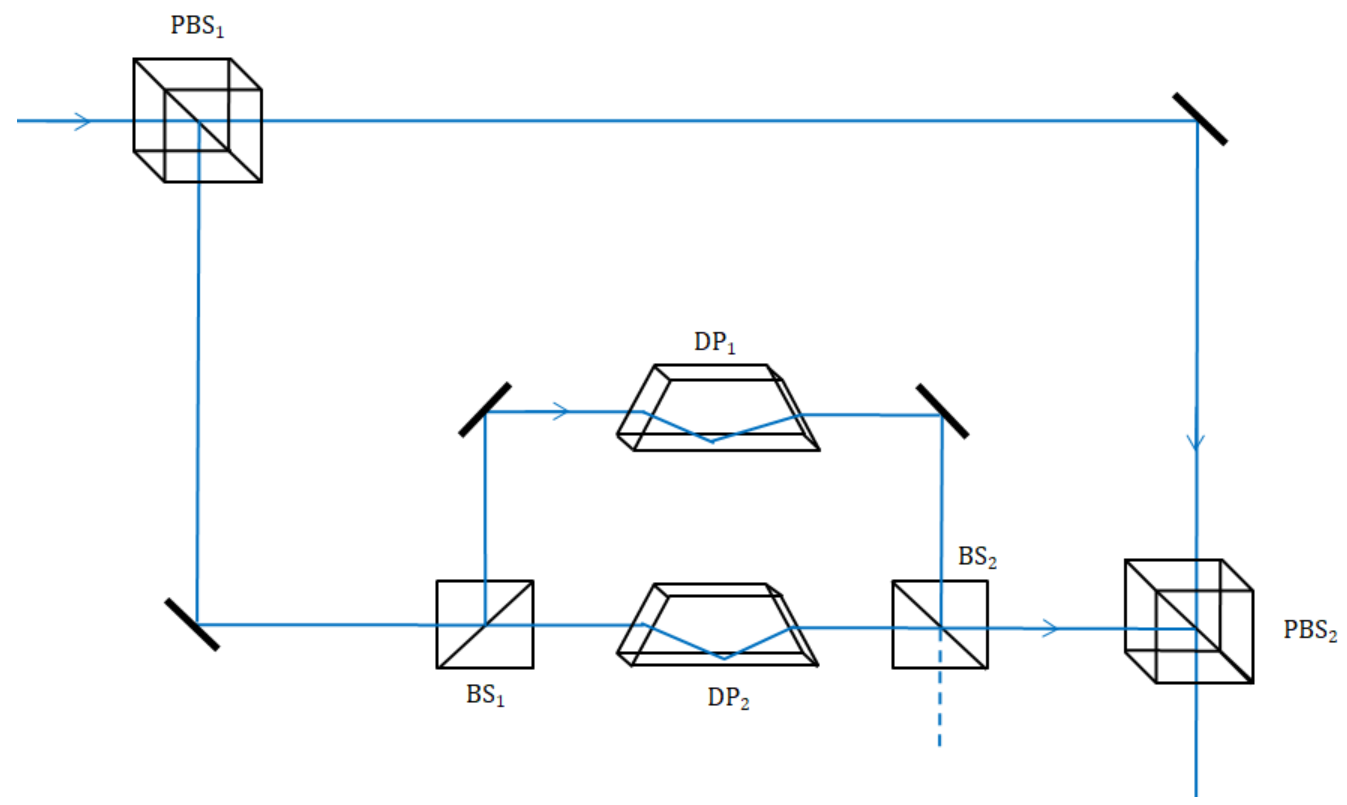

FIG. 1: Apparatus for the realisation of a Hyper-CPHASE over polarisation and orbital angular degrees of freedom. Here $P B S$ - polarising beam splitter, $D P$ - Dove Prism, $B S$ - Beam Splitter. Applying beam splitter operation to the light beam, the photonic quantum state evolves through the two arms of the OAM-flip interferometer with a phase of $\frac{\pi}{2}$. Here, $D P_{2}$ is rotated by an angle $\frac{\pi}{2}$ to implement the logic Z-gate. One of the mirrors in the OAM-flip interferometer module is piezeo-controlled to allow for fine-adjustments

In Figure 1, the two branches have orthogonal polarisation states, with a partial phase plate in one of the branches. Since we have control on the polarisation in each branch, upto global arbitrary phases, the action of the phase plate is on a specific OAM state (either $\left|E_{(0)}\right\rangle$ or $\left.\left|E_{(1)}\right\rangle\right)$. If we allow the $|1\rangle$ state to enter the lower branch in the apparatus in Figure 1, we have the following operation

$$
\left|\psi_{i n}\right\rangle \stackrel{P B S_{1}+P P}{\longrightarrow}|0\rangle\left|E_{(0)}\right\rangle+|1\rangle U_{P P}\left|E_{(1)}\right\rangle
$$

where $U_{P P}$ denotes the operation of the phase plate. This operation is implemented using an interferometer setup, as shown in Figure 1. The dove prism in the second branch introduces an OAM-dependent phase $e^{\iota q \pi}$ where $q$ corresponds to the OAM state $|q\rangle$. In the apparatus in Figure 1, the even states acquire a phase of $e^{i 2 k \pi}$ which effectively leaves the state unchanged, while the odd states acquire a phase of $e^{i(2 k+1) \pi}$ which brings a negative sign to these odd states. Therefore, we have $U_{P P}|E\rangle=|E\rangle$ for OAM even state and $P P|E\rangle=-|E\rangle$ for OAM odd state. We can also realise an OAM-controlled Hyper-CPHASE gate using the OAM-interferometer but with a relative angle between the dove prisms being zero. In one of the branches, we can use an optical phase shifter in the form of a Half Wave Plate (HWP) to implement a relative phase between the fundamental polarisation qubits.
The implementation of the Hyper-CPHASE gives us an idea about how to implement a hyper-conditional quantum gate.

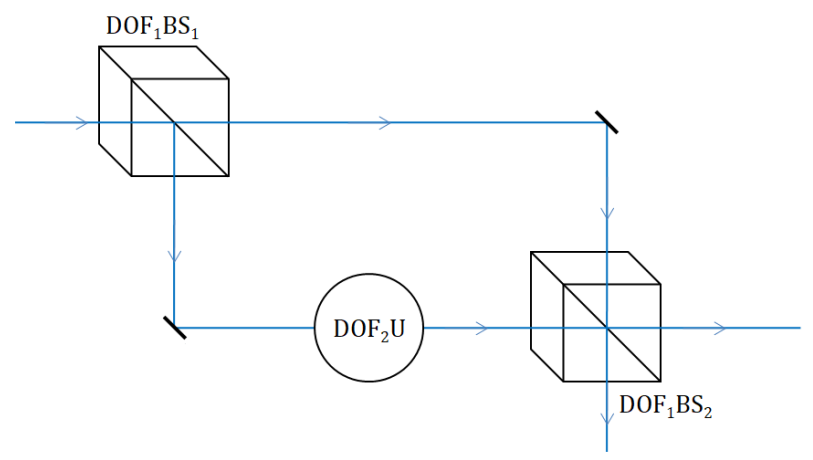

FIG. 2: Scheme for an arbitrary Hyper-Conditional Gate. Here we have two degrees of freedom: $\mathrm{DOF}_{1}$ and $\mathrm{DOF}_{2}$, with the first as control and second as target. $\mathrm{DOF}_{1} \mathrm{BS}_{i}$ represents the $i^{\text {th }}$ beamsplitter that discriminates states based on the states of the first degree of freedom. $\mathrm{DOF}_{2} \mathrm{U}$ is the operation being performed on the second qubit

For a hyper-controlled gate, we focus on the control degree of freedom $\left(\mathrm{DOF}_{c}\right)$ and target degree of freedom 
$\left(\mathrm{DOF}_{t}\right)$. We can define a general state as

$$
\left|S D_{1} D_{2}\right\rangle=\left(\begin{array}{c}
s_{1} \\
s_{2}
\end{array}\right) \otimes\left(\begin{array}{c}
d_{1}^{(1)} \\
d_{2}^{(1)}
\end{array}\right) \otimes\left(\begin{array}{c}
d_{1}^{(2)} \\
d_{2}^{(2)}
\end{array}\right)=\left(\begin{array}{c}
s_{1} d_{1}^{(1)} d_{1}^{(2)} \\
s_{1} d_{1}^{(1)} d_{2}^{(2)} \\
s_{1} d_{2}^{(1)} d_{1}^{(2)} \\
s_{1} d_{2}^{(1)} d_{2}^{(2)} \\
s_{2} d_{1}^{(1)} d_{1}^{(2)} \\
s_{2} d_{1}^{(1)} d_{2}^{(2)} \\
s_{2} d_{2}^{(1)} d_{1}^{(2)} \\
s_{2} d_{2}^{(1)} d_{2}^{(2)}
\end{array}\right)
$$

For the control, we use the control qubit and the spatial channels to implement the control-element of the scheme. If we define the initial state reflectance $(r)$ and transmittance $(t)$ within a DOF-based beam-splitter matrix, we can define the composite control-operator $C$

$$
C=\left(\begin{array}{cccccccc}
t_{1} & 0 & 0 & 0 & \iota r_{1} & 0 & 0 & 0 \\
0 & t_{1} & 0 & 0 & 0 & \iota r_{1} & 0 & 0 \\
0 & 0 & t_{2} & 0 & 0 & 0 & \iota r_{2} & 0 \\
0 & 0 & 0 & t_{2} & 0 & 0 & 0 & \iota r_{2} \\
\iota r_{1} & 0 & 0 & 0 & t_{1} & 0 & 0 & 0 \\
0 & \iota r_{1} & 0 & 0 & 0 & t_{1} & 0 & 0 \\
0 & 0 & \iota r_{2} & 0 & 0 & 0 & t_{2} & 0 \\
0 & 0 & 0 & \iota r_{2} & 0 & 0 & 0 & t_{2}
\end{array}\right)
$$

where $t_{i}$ and $r_{i}$ are the transmittance and reflectance respectively for the $i^{\text {th }}$ state of the control (DOF) qubit. $C^{\prime}$ is the same operation with $t_{i} \leftrightarrow \iota r_{i}$. The target operation $(T)$ can be an arbitrary single qubit operation $U$. Seeing the sequence of operations in Figure 2 and accounting for the directions of the beams in different sections of the circuit, we see that the composite operation is $O=C .(I \otimes(I \oplus U)) . C^{\prime}$, whose matrix form is

$$
\left.\begin{array}{cccc}
t_{1}^{2}-r_{1}^{2} & 0 & 0 & 0 \\
0 & t_{1}^{2}-r_{1}^{2} & 0 & 0 \\
0 & 0 & U_{11}\left(t_{2}^{2}-r_{2}^{2}\right) & U_{12}\left(t_{2}^{2}-r_{2}^{2}\right) \\
0 & 0 & U_{21}\left(t_{2}^{2}-r_{2}^{2}\right) & U_{22}\left(t_{2}^{2}-r_{2}^{2}\right) \\
2 \iota t_{1} r_{1} & 0 & 0 & 0 \\
0 & 2 \iota t_{1} r_{1} & 0 & 0 \\
0 & 0 & 2 \iota t_{2} r_{2} U_{11} & 2 \iota t_{2} r_{2} U_{12} \\
0 & 0 & 2 \iota t_{2} r_{2} U_{21} & 2 \iota t_{2} r_{2} U_{22}
\end{array}\right)
$$

If we consider the perfect $\mathrm{DOF}_{1}$-based beam splitter is perfect with $t_{1}=i r_{2}=1$ and $t_{2}=i r_{1}=0$ and the operator for $\mathrm{DOF}_{2}$ to have $U_{11}=1, U_{12}=0, U_{21}=0$ and $U_{22}=-1$, giving us $\sigma_{x} \otimes \mathrm{CPHASE}$. This effectively implements the CPHASE gate between the two degrees of freedom $\mathrm{DOF}_{1}$ and $\mathrm{DOF}_{2}$. While the polarising beam splitter is well known, beam splitters based on other degrees of freedom, such as orbital angular momentum have been analysed and used recently [28]. The proposed scheme works for any degree of freedom wherein experimental resources for mode sorting and mode shifting are available. Instead of a DOF-based beam splitter, we can also use a probabilistic version of this scheme with a beam splitter followed by DOF-filters that project the states in the relevant DOF to either $|0\rangle$ or $|1\rangle$. Quantum Non-Demolition (QND) Measurement can not only be used to look at the values of the various degrees of freedom for a given photonic qubit without destroying its state but has recently also been used for teleporting multiple degrees of freedom of a photon [29].

While a generalised physical model for all DOFbased sorters is not present at the moment, we can look at the relevant optical elements for an exhaustive set of all degrees of freedom of a photon [30] - polarization, spatial (OAM) mode, and energy time (bin):

\begin{tabular}{|c|c|c|}
\hline Degree of Freedom & DOF-BS & Gates \\
\hline Polarisation & Polarising Beam Splitter & Universal [8] \\
\hline OAM & OAM-Sorter & Universal [31] \\
\hline Time/Frequency & Dichroic Mirror & Universal [32] \\
\hline
\end{tabular}

TABLE I: Some degrees of Freedom of a photonic qubit, along with associated characteristic DOF-BS or sorter and universality of gates for the realisation of arbitrary Hyper-conditional gates

\section{CONCLUSION}

In this paper, a hyper-CPHASE gate using the polarisation and orbital angular momentum degrees of freedom for single-photon two-qubit quantum logic was proposed. As this is important for the realisation of quantum cluster states and graph networks using transverse degrees of freedom, the proposed model and scheme has applications in various quantum information processing tasks and protocols. A generalisation of the proposed scheme, for an arbitrary hyper-conditional gate was proposed. This proposal can pave the path to optimal generation and implementation of arbitrary condition-gates across various internal degrees of freedom of photonic qubits. 
[1] D. P. DiVincenzo, Physical Review A 51, 1015 (1995).

[2] D. Gottesman and I. L. Chuang, Nature 402, 390 (1999).

[3] J. L. O'brien, A. Furusawa, and J. Vučković, Nature Photonics 3, 687 (2009).

[4] L.-M. Duan and H. Kimble, Physical review letters 92, 127902 (2004).

[5] K. Azuma, K. Tamaki, and H.-K. Lo, Nature communications 6, 6787 (2015).

[6] K. Chen, C.-M. Li, Q. Zhang, Y.-A. Chen, A. Goebel, S. Chen, A. Mair, and J.-W. Pan, Physical review letters 99, 120503 (2007).

[7] R. Prevedel, P. Walther, F. Tiefenbacher, P. Böhi, R. Kaltenbaek, T. Jennewein, and A. Zeilinger, Nature 445, 65 (2007).

[8] E. Knill, R. Laflamme, and G. J. Milburn, nature 409, 46 (2001).

[9] T. C. Ralph, N. K. Langford, T. Bell, and A. White, Physical Review A 65, 062324 (2002).

[10] J. L. O'Brien, G. J. Pryde, A. G. White, T. C. Ralph, and D. Branning, Nature 426, 264 (2003).

[11] S. Gasparoni, J.-W. Pan, P. Walther, T. Rudolph, and A. Zeilinger, Physical review letters 93, 020504 (2004).

[12] F.-G. Deng, B.-C. Ren, and X.-H. Li, Science Bulletin 62, 46 (2017).

[13] G. Vallone, G. Donati, R. Ceccarelli, and P. Mataloni, Physical Review A 81, 052301 (2010).

[14] I. Schwartz, D. Cogan, E. R. Schmidgall, Y. Don, L. Gantz, O. Kenneth, N. H. Lindner, and D. Gershoni, Science 354, 434 (2016).

[15] M. Kues, C. Reimer, S. Sciara, P. Roztocki, M. Islam, L. R. Cortés, Y. Zhang, B. Fischer, S. Loranger, R. Kashyap, et al., in Quantum Information and Measurement (Optical Society of America, 2019) pp. S2C-3.

[16] S. Yokoyama, R. Ukai, S. C. Armstrong, C. Sornphiphatphong, T. Kaji, S. Suzuki, J.-i. Yoshikawa, H. Yonezawa, N. C. Menicucci, and A. Furusawa, Nature Photonics 7, $982(2013)$.
[17] M. V. Larsen, X. Guo, C. R. Breum, J. S. NeergaardNielsen, and U. L. Andersen, Science 366, 369 (2019).

[18] R. Raussendorf and H. J. Briegel, Physical Review Letters 86, 5188 (2001).

[19] R. Raussendorf, J. Harrington, and K. Goyal, Annals of physics 321, 2242 (2006).

[20] G. Vallone, E. Pomarico, P. Mataloni, F. De Martini, and V. Berardi, Physical review letters 98, 180502 (2007).

[21] X.-L. Wang, Y.-H. Luo, H.-L. Huang, M.-C. Chen, Z.-E. Su, C. Liu, C. Chen, W. Li, Y.-Q. Fang, X. Jiang, et al., Physical review letters 120, 260502 (2018).

[22] X. Zou, K. Li, and G. Guo, Physical Review A 74, 044305 (2006).

[23] K. Lemr, A. Černoch, J. Soubusta, K. Kieling, J. Eisert, and M. Dušek, Physical review letters 106, 013602 (2011).

[24] S. Das, A. Grankin, I. Iakoupov, E. Brion, J. Borregaard, R. Boddeda, I. Usmani, A. Ourjoumtsev, P. Grangier, and A. S. Sørensen, Physical Review A 93, 040303 (2016).

[25] Y.-F. Xiao, X.-B. Zou, and G.-C. Guo, Physical Review A 75, 054303 (2007).

[26] I. Fushman, D. Englund, A. Faraon, N. Stoltz, P. Petroff, and J. Vučković, science 320, 769 (2008).

[27] L.-B. Chen and W. Yang, Laser Physics Letters 11, 105201 (2014).

[28] J. Leach, M. J. Padgett, S. M. Barnett, S. Franke-Arnold, and J. Courtial, Phys. Rev. Lett. 88, 257901 (2002).

[29] X.-L. Wang, X.-D. Cai, Z.-E. Su, M.-C. Chen, D. Wu, L. Li, N.-L. Liu, C.-Y. Lu, and J.-W. Pan, Nature 518 , $516(2015)$.

[30] J. T. Barreiro, N. K. Langford, N. A. Peters, and P. G. Kwiat, Physical review letters 95, 260501 (2005).

[31] J. C. García-Escartín and P. Chamorro-Posada, Journal of Optics 13, 064022 (2011).

[32] H.-H. Lu, J. M. Lukens, B. P. Williams, P. Imany, N. A. Peters, A. M. Weiner, and P. Lougovski, npj Quantum Information 5, 1 (2019). 